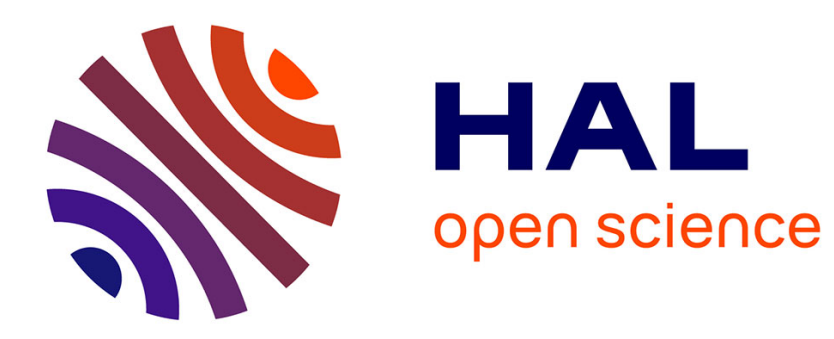

\title{
Position estimation from direction or range measurements
}

\author{
Tarek Hamel, Claude Samson
}

\section{To cite this version:}

Tarek Hamel, Claude Samson. Position estimation from direction or range measurements. Automatica, 2017, 82, pp.137-144. 10.1016/j.automatica.2017.04.045 . hal-01629173

\section{HAL Id: hal-01629173 \\ https://hal.science/hal-01629173}

Submitted on 6 Nov 2017

HAL is a multi-disciplinary open access archive for the deposit and dissemination of scientific research documents, whether they are published or not. The documents may come from teaching and research institutions in France or abroad, or from public or private research centers.
L'archive ouverte pluridisciplinaire HAL, est destinée au dépôt et à la diffusion de documents scientifiques de niveau recherche, publiés ou non, émanant des établissements d'enseignement et de recherche français ou étrangers, des laboratoires publics ou privés. 


\title{
Position estimation from direction or range measurements
}

\author{
Tarek Hamel $^{\text {a }}$, Claude Samson ${ }^{\text {b }}$ \\ ${ }^{a}$ Université Côte d'Azur, CNRS, I3S, France \\ ${ }^{\mathrm{b}}$ Université Côte d'Azur, INRIA, CNRS, I3S, France
}

\begin{abstract}
This paper revisits the problems of estimating the position of an object moving in $n(\geq 2)$-dimensional Euclidean space using velocity measurements and either direction or range measurements of one or multiple source points. The proposed solutions exploit the Continuous Riccati Equation (CRE) to calculate observer gains yielding global uniform exponential stability of zero estimation errors, also when the measured velocity is biased by an unknown constant vector or when direction measurements are corrupted by an unknown constant bias.

With respect to prior contributions on these subjects they provide a coherent generalization of existing solutions with the preoccupation of pointing out general and explicit persistent excitation (p.e.) conditions whose satisfaction ensures uniform exponential stability of the observers.
\end{abstract}

Key words: position estimation; Riccati observers; linear time-varying systems; persistent excitation; observability.

\section{Introduction}

The general problem of estimating the position, or the complete pose (position and orientation), of a body relatively to a certain spatial frame is central for a multitude of applications. The present paper focuses on the sole estimation of the body position using velocity measurements and either direction or range measurements of one or multiple source points. This corresponds to applications for which the body's attitude is either of lesser importance or is estimated by using other sensing modalities. In this case, iterative (gradient search) methods are all the more interesting that their domain of convergence can be global. Another advantage of iterative methods is that they are naturally suited to handle the non-static case, i.e. when either the body or the point source(s) move(s), by using on-line the extra data and information resulting from motion. In particular, the observation of a single source point may be sufficient in this case, provided that the body motion regularly grants a sufficient amount of "observability". In the case of direction measurements this possibility has been studied recently in [?] by rendering the output equation linear in the state and applying Kalman filtering. A different solution not resorting on the use of a CRE is proposed in [?]. Discrete-time extensions of

Email addresses: thamel@i3s.unice.fr (Tarek Hamel), claude.samson@inria.fr, csamson@i3s.unice.fr (Claude Samson). the solution derived in [?] to the case of multiple source points are derived in [?]. The present paper borrows solutions from these latter references, regroups them in a single general framework, and complements them with the characterization of new general observability conditions whose satisfaction grants good-conditioning and global exponential stability to the proposed estimators. Global Navigation Satellite Systems (GNSS), and the American Global Positioning System (GPS) [?] in particular, have familiarized the larger public with the problem of body position estimation from source points distance (or range) measurements. In the static case (motionless body) three point sources (satellites) are required to algebraically calculate a finite number (equal to two) of theoretical solutions, with an extra source point (non-coplanar with the other points) needed to eliminate the non-physical solution and overcome the problem of desynchronized clocks resulting in constant range measurement bias. Studies of the non-static case are much less numerous and more recent. The observer solutions proposed in the present paper are inspired from the pioneering works of Batista and al. [?,?,?] on the subject who exploit the possibility of linearising the estimation problem via state augmentation. This possibility is also used here, but with some noticeable differences concerning in particular the augmented state definition and the formulation of persistent excitation (p.e.) conditions ensuring uniform exponential stability (not just convergence) of the observers. For instance, 
preventing the state dimension from growing with the number of source points yields simpler observers and reduced computational weight. From our perspective, the p.e. conditions here proposed are also more natural and easily interpreted, and better assess of the gradual observability increase resulting from using more source points. It is here assumed that the object's velocity is available to measurement, except for a possible unknown constant bias. A way to avoid this assumption via filtering is proposed in [?].

For five decades, Kalman filters for linear systems, and their extensions to non-linear systems known as Extended Kalman Filters (EKF), have consistently grown in popularity near engineers with various backgrounds (signal processing, artificial vision, robotics,...) to address a multitude of iterative state estimation problems involving additive "noise" upon the state and/or the measurements. The optimality of these filters in a stochastic framework under specific noise conditions and assumptions, and their direct applicability to Linear Time-Varying (LTV) systems, have undoubtedly contributed to this popularity. It is however important to keep in mind, or to recall, that the stability and robustness properties associated with them, i.e. features that supersede conditional stochastic optimality in practice, are not related to stochastic issues. They result from properties of the associated deterministic continuoustime (or discrete-time, depending on the chosen computational framework) Riccati equation that underlies a (locally) convex estimation error index (or Lyapunov function) and a way of forming recursive estimation algorithms that uniformly decrease this index exponentially (under adequate observability conditions). With this perspective, Kalman filters belong to the (slightly) larger set of Riccati observers that we intentionally derive here in a deterministic framework, knowing that a complementary stochastic interpretation may be useful to subsequently tune the Riccati equation parameters and observer gains. This tuning issue is important for practical purposes and deserves to be studied in its own right. However, it is out of the present paper's scope and is thus not pursued further here. We also believe that, by contrast with standard Kalman filter derivations performed in a stochastic framework, the deterministic approach here considered allows one to more directly comprehend how the system observability properties (uniform observability resulting from persistent excitation, in particular) are related to the good conditioning of the Riccati equation solutions and to the observer's performance (the rate of convergence to zero of the estimation errors, in particular) via a Lyapunov analysis. The research themes addressed in the present paper are not new, nor are the basic conceptual tools (Riccati equation, Lyapunov stability, uniform observability and persistent excitation,...) used to derive the propose observers. However, we believe that the reported global approach to the problems, the proposed observers derived for both direction measurements and range measurements, in $n(\geq 2)$-dimensional Euclidean space with an arbitrary number of source points, and the worked out p.e. conditions ensuring uniform exponential stability of these observers are original.

The paper is organized along six sections. Following the present introduction, Section 2 recalls basic observability concepts and central properties of the CRE, complemented with technical results used for stability and convergence analysis of the observers. Direction measurements and range measurements cases are treated in Sections 3 and 4 respectively. Illustrative simulations results are presented in Section 5 , followed by a short section 6 of concluding remarks. The proofs of several technical results are reported in the Appendix.

\section{Recalls}

This section provides the reader with a short selfcontained overview of basic observability concepts and of state observers whose gains are calculated from solutions to the Continuous Riccati Equation (CRE). This overview is also an opportunity to recall Lyapunov function candidates associated with these observers for stability and convergence analysis.

Throughout the paper the following notation is used:

- $A(t), B(t), C(t)$ are finite-dimensional matrix-valued functions depending on time. They are continuous, bounded, and $r(\geq 0)$ times differentiable with bounded derivatives, with $r$ specified (sometimes implicitly) in subsequent developments.

- The abbreviation p.s.d. (resp. p.d.) is used to denote positive semidefinite (resp. positive definite) square matrices that are also symmetric. Identity matrices are p.d. matrices and denoted as $I_{d}$ independently of their dimensions.

- $Q(t)$ and $V(t)$ are p.s.d. finite-dimensional matrixvalued functions of time. They are also continuous and bounded. When no specific indication is provided in the text these matrix-valued functions are chosen strictly positive and greater than $\epsilon I_{d}$ with $\epsilon>0$.

- The infimum (resp. supremum) over time of the smallest (resp. largest) eigenvalue of a p.s.d matrix-valued function $P(t)$ is denoted as $p_{m}$ (resp. $p_{M}$ ). For the matrix-valued function $V(t)$ these infimum and supremum values are accordingly denoted as $v_{m}$ and $v_{M}$.

\subsection{Observability definitions and conditions}

Consider a generic linear time-varying (LTV) system

$$
\left\{\begin{array}{l}
\dot{x}=A(t) x+B(t) u \\
y=C(t) x
\end{array}\right.
$$

with $x \in \mathbb{R}^{n}$ the system state, $u \in \mathbb{R}^{s}$ the system input, and $y \in \mathbb{R}^{m}$ the system output. The following definitions and properties of observability associated with this system are borrowed from [?] and [?].

\section{Definition 2.1 (instantaneous observability)}

System (1) is instantaneously observable if $\forall t, x(t)$ can be calculated from the input $u(t)$, the output $y(t)$, and the time-derivatives $u^{(k)}(t), y^{(k)}(t), k \in \mathbb{N}$. 
Lemma 2.2 Define the observation space at the timeinstant $t$ as the space generated by

$$
\mathcal{O}(t):=\left(\begin{array}{c}
N_{0}(t) \\
N_{1}(t) \\
\vdots
\end{array}\right)
$$

with $N_{0}=C, N_{k+1}=N_{k} A+\dot{N}_{k}, k=1, \ldots$ Then System (1) is instantaneously observable if $\operatorname{rank}(\mathcal{O}(t))=n$.

Theorem 2.3 (uniform observability) System (1) is uniformly observable if there exist $\delta>0, \mu>0$ such that $\forall t \geq 0$ :

$W(t, t+\delta):=\frac{1}{\delta} \int_{t}^{t+\delta} \Phi^{\top}(s, t) C^{\top}(s) C(s) \Phi(s, t) d s \geq \mu I_{d}>0$

with $\Phi(t, s)$ the transition matrix associated with $A(t)$, i.e. such that $\frac{d}{d t} \Phi(t, s)=A(t) \Phi(t, s)$ with $\Phi(t, t)=I_{d}$.

The matrix valued-function $W(t, t+\delta)$ is called the $o b$ servability Gramian of System (1). This definition of uniform observability is different from other definitions proposed in the literature, e.g. [?] or [?]. What matters here is that the condition (2) is the one needed to establish good conditioning and exponential stability of the estimators derived in the present paper. The following lemma, taken from [?], gives a sufficient condition for uniform observability in terms of the properties of the matrices $A(t)$ and $C(t)$ and their time-derivatives:

Lemma 2.4 If there exists a matrix-valued function $M($.$) of dimension (p \times n)(p \geq 1)$ composed of row vectors of $N_{0}(),. N_{1}(),. \ldots$, such that for some (strictly) positive numbers $(\bar{\delta}, \bar{\mu})$ and $\forall t \geq 0$

$$
\frac{1}{\bar{\delta}} \int_{t}^{t+\bar{\delta}} M^{\top}(s) M(s) d s \geq \bar{\mu} I_{d}>0
$$

then the observability Gramian of System (1) satisfies the condition (2).

\subsection{Riccati observers}

We here call Riccati observer any observer of System (1) of the form

$$
\dot{\hat{x}}=A(t) \hat{x}+B(t) u+K(t)(y-C(t) \hat{x}) ; \hat{x}(0) \in \mathbb{R}^{n}
$$

with the observer gain given by

$$
K(t)=k(t) P(t) C^{\top}(t) Q(t) ; k(t) \geq 0.5
$$

where $P(t)$ is the solution to the so-called Continuous Riccati Equation (CRE)

$$
\dot{P}=A(t) P+P A(t)^{\top}-P C^{\top}(t) Q(t) C(t) P+V(t)
$$

with $P(0)$ any p.d. matrix and $Q(t), V(t)$ p.s.d. matrices that have to be specified. Note that the optimal Kalman gain in the stochastic setting where the matrices $V(t)$ and $Q^{-1}(t)$ are interpreted as covariance matrices of additive noise on the system state and output is obtained by taking $k(t)=1$. Note also that stochastic Kalman filtering does not cover the case of a p.s.d. matrix $Q(t)$. The constant solution $P(t)=I_{d}(\forall t)$ obtained when $A(t)$ is skew-symmetric and by setting $V(t)=C^{\top}(t) Q(t) C(t)$ is also seldom considered whereas it avoids any calculation and is useful to make the connection between Riccati observers and a number of Lyapunov-based designs proposed in the literature.

Let us now quickly recall how the stability and convergence properties of a Riccati observer is directly related to the properties of the solution $P(t)$ to the CRE. Define the estimation error $\tilde{x}:=x-\hat{x}$, from (1) and (4) one obtains the error equation

$$
\dot{\tilde{x}}=(A(t)-K(t) C(t)) \tilde{x}
$$

Assume (for the time being) that $P(t)$, which is a symmetric matrix by construction, is well defined on $\mathbb{R}^{+}$and is p.d., so that its inverse is also well defined and p.d., and consider the candidate Lyapunov function $\mathcal{V}(t)=$ $\tilde{x}^{\top}(t) P^{-1}(t) \tilde{x}(t)$. Then using the fact that the timederivative of $P^{-1}$ satisfies the relation $\dot{P}^{-1}=-P^{-1} A(t)-A^{\top}(t) P^{-1}+C^{\top}(t) Q(t) C(t)-P^{-1} V(t) P^{-1}$

and using (5) and (7), one easily verifies that the timederivative of $\mathcal{V}(t)$ is given by

$$
\begin{aligned}
\dot{\mathcal{V}} & =-\tilde{x}^{\top}\left((2 k(t)-1) C^{\top}(t) Q(t) C(t)+P^{-1} V(t) P^{-1}\right) \tilde{x} \\
& \leq-\tilde{x}^{\top} P^{-1} V(t) P^{-1} \tilde{x} \leq-\frac{p_{m}}{p_{M}^{2}} v_{m} \mathcal{V} \quad(\leq 0)
\end{aligned}
$$

so that $\mathcal{V}(t) \leq \mathcal{V}(0) \exp \left(-\frac{p_{m}}{p_{M}^{2}} v_{m} t\right)$. To conclude that $\tilde{x}=0$ is globally uniformly exponentially stable it suffices to choose $V(t)>v_{m} I_{d}$ with $v_{m}>0$ and to show that $P(t)$ i) is always well-defined, ii) that it is p.d., and -most importantly- iii) that it is well conditioned in the sense that $p_{m}$ is strictly positive and $p_{M}$ is finite so that the ratio $\frac{P_{M}^{2}}{p_{m}}$ is bounded. Since the inverse of this ratio essentially determines the exponential rate of convergence of the estimation errors to zero, it is of interest to know bounds of $p_{m}$ and $p_{M}$ in relation to the "amount" of observability, itself closely related to the amount of persistent excitation. Such bounds are derived in Appendix A.1, with a lower bound of $p_{M}$ calculated from an expression derived in [?]. The central issue of boundedness and good conditioning of $P(t)$ brings us to recall classical, and also point out less known, results concerning the CRE.

\subsection{Properties of the Continuous Riccati Equation}

The first results concerns the existence of the solutions to the CRE for $t \in[0,+\infty)$.

Lemma 2.5 If $P(0)$ is p.d. and $Q(t)$ and $V(t)$ are p.s.d, then $P(t)$ is $p . d$. and well defined on $[0,+\infty)$.

See a proof in [?], for instance. Now, to ensure boundedness and good-conditioning of the solution $P(t)$ to the CRE one has to impose other conditions upon the terms entering the equation. Sufficient conditions are pointed out in the next lemma.

Lemma 2.6 Define:

$$
W_{V}(t, t+\delta):=\frac{1}{\delta} \int_{t}^{t+\delta} \Phi(t, s) V(s) \Phi^{\top}(t, s) d s
$$


and

$W_{Q}(t, t+\delta):=\frac{1}{\delta} \int_{t}^{t+\delta} \Phi^{\top}(s, t) C^{\top}(s) Q(s) C(s) \Phi(s, t) d s$

If there exist (strictly) positive numbers $\delta, \mu_{v}$, and $\mu_{q}$ such that $W_{V}(t, t+\delta) \geq \mu_{v} I_{d}$ and $W_{Q}(t, t+\delta) \geq \mu_{q} I_{d}$, $\forall t$, then the solution $P(t)$ to the CRE (6) is bounded and well-conditioned in the sense that $0<p_{m} \leq p_{M}<\infty$.

A proof of this result is given in [?] where lower and upper bounds of $P(t)$ are also derived. The matrix $Q(t)$ is in fact assumed p.d. because the inverse of $Q$ is (for technical convenience) used in the proof. However, it is simple to verify that the proposed bounds for $P(t)$ do not depend on the smallest eigenvalue of $Q(t)$, so that these bounds are also valid when $Q(t)$ is only p.s.d.

Note that if $Q(t) \geq \epsilon I_{d}>0$ and the observability Gramian $W$ satisfies the positivity condition (2), then the Riccati observability Gramian $W_{Q}$ satisfies a similar condition. This is just a consequence of that $W_{Q}(t, t+\delta) \geq \lambda_{\min }(Q(t)) W(t, t+\delta)$. A technical lemma used to derive a p.e. condition for the direction-based position observers proposed in the next section is as follows

Lemma 2.7 Let

- $A$ and $H$ denote two constant real matrices of respective dimensions $n \times n$ and $m \times n$ and such that

(1) the pair $(A, H)$ is (Kalman) observable, i.e. $\operatorname{rank}\left[H, H A, \ldots, H A^{n-1}\right]=n$.

(2) all eigenvalues of $A$ are real.

- $\Sigma(t)$ denote a bounded $(m \times m)$-dimensional p.s.d matrix depending on time and such that for some $\delta>0$

$$
\forall t: \frac{1}{\delta} \int_{t}^{t+\delta} \Sigma(s) d s \geq \bar{\epsilon} I_{d}>0 .
$$

- $W_{A, H}^{\Sigma}(t, t+\bar{\delta}):=\frac{1}{\delta} \int_{t}^{t+\bar{\delta}} \Phi^{\top}(s, t) H^{\top} \Sigma(s) H \Phi(s, t) d s$ with $\Phi(s, t)=\exp (A(s-t))$.

Then there exists $\mu>0$ and $\bar{\delta}>0$ such that

$$
\forall t: W_{A, H}^{\Sigma}(t, t+\bar{\delta})>\mu .
$$

The proof of this lemma is given in Appendix A.2.

\section{Position estimation from directions}

Given the coordinates $z_{i}(i \in\{1, \ldots, l\})$ of $l$ source points expressed in some fixed frame, the problem consists in estimating the position $x$ of a body (or object) with respect to (w.r.t.) this frame knowing its velocity $u$ and by using the measurement of the $l$ directions $y_{i}:=\left(x-z_{i}\right) /\left|x-z_{i}\right|$ from the source points to the body. For the sake of generality, and because this may be useful in practice, we also treat the case when the measured velocity is biased by an initially unknown constant vector $a$. In practice, $x$ will be a two-dimensional vector of coordinates (in the 2D, or planar, case) or a threedimensional vector of coordinates (in the $3 \mathrm{D}$, or spatial, case). Again for the sake of generality, we assume that $x \in \mathbb{R}^{n}$, with $n \geq 2$.

Consider the projection matrix operator $\Pi_{\sigma}:=I_{d}-$ $\sigma \sigma^{\top}$ with $\sigma \in \mathbb{R}^{n}$ and such that $|\sigma|=1$ (i.e. $\sigma \in$ $\left.S^{n-1}\right)$. Setting $X:=\left[x^{\top}, a^{\top}\right]^{\top}, \bar{u}:=\left[u^{\top}, 0_{1 \times n}\right]^{\top}$, and $y=\left[\left(\Pi_{y_{1}(t)} z_{1}\right)^{\top}, \ldots,\left(\Pi_{y_{l}(t)} z_{l}\right)^{\top}\right]^{\top}$, one obtains the system

$$
\left\{\begin{array}{l}
\dot{X}=A X+\bar{u} \\
y=C(t) X
\end{array}\right.
$$

with

$$
\begin{aligned}
A & =\left[\begin{array}{cc}
0_{n \times n} & I_{n \times n} \\
0_{n \times n} & 0_{n \times n}
\end{array}\right] \\
C(t) & =\operatorname{blockdiag}\left(\Pi_{y_{1}(t)}, \ldots, \Pi_{y_{l}(t)}\right) \bar{C} \\
\bar{C} & =\left[\begin{array}{cc}
I_{n \times n} & 0_{n \times n} \\
\vdots & \\
I_{n \times n} & 0_{n \times n}
\end{array}\right] \text { with } \operatorname{dim}(\bar{C})=\ln \times 2 n
\end{aligned}
$$

A Riccati observer associated with this system is

$$
\begin{aligned}
& \dot{\hat{X}}=A \hat{X}+\bar{u}+K(t)(y-C(t) \hat{X}) \\
& K(t)=k(t) P(t) C^{\top}(t) Q(t) \quad(k(t) \geq 0.5) \\
& \dot{P} \quad=A P+P A^{\top}-P C^{\top}(t) Q(t) C(t) P+V(t)
\end{aligned}
$$

with $\hat{X}:=\left[\hat{x}^{\top}, \hat{a}^{\top}\right]^{\top}$. Setting

$$
Q(t)=\operatorname{blockdiag}\left(Q_{11}(t), \ldots, Q_{l l}(t)\right)
$$

with $Q_{i i}(t)>\epsilon I_{n \times n}(i \in\{1, \ldots, l\})$ for some $\epsilon>0^{1}$, one easily verifies that this observer can also be written as

$$
\left\{\begin{array}{l}
\dot{\hat{x}}=u+\hat{a}-k(t) P_{11}(t)\left(\sum_{i=1}^{l} \Pi_{y_{i}(t)} Q_{i i}(t)\left(\hat{x}-z_{i}\right)\right) \\
\dot{\hat{a}}=-k(t) P_{21}(t)\left(\sum_{i=1}^{l} \Pi_{y_{i}(t)} Q_{i i}(t)\left(\hat{x}-z_{i}\right)\right)
\end{array}\right.
$$

To prove that this observer is globally uniformly exponentially stable it suffices to show that the solution $P(t)$ to the CRE is bounded and well conditioned and, to this end, to show that, for some $\delta>0$ and $\forall t$, the corresponding observability Gramian $W_{Q}(t, t+\delta)$ is larger than some positive number. The following lemma provides a p.e. condition whose satisfaction ensures this latter property.

Lemma 3.1 Define $\Delta(t):=\sum_{i=1}^{l} \Pi_{y_{i}(t)}$. If there exists $\delta>0$ and $\mu>0$ such that $\forall t$ the following p.e. condition is satisfied

$$
\forall t \geq 0: \frac{1}{\delta} \int_{t}^{t+\delta} \Delta(s) d s>\mu
$$

then the Riccati observer (17)globally uniformly exponentially stabilizes $\tilde{X}=0$.

The proof of this lemma relies on the application of the technical Lemma 2.7 and is given in Appendix A.3. Remarks:

- In the case of a single source point one easily verifies that the p.e. condition (18) is essentially equivalent to

${ }^{1}$ one can also use the p.s.d. matrix $Q_{i i}(t)=\gamma_{i} \Pi_{y_{i}(t)}\left(\gamma_{i}>\right.$ $0)$ that yields the same Riccati equation as $Q_{i i}(t) \stackrel{\gamma_{i}}{=} \gamma_{i} I_{n \times n}$ 
requiring that $\left|\dot{y}_{1}(t)\right|$ is regularly larger than a positive number.

- This condition also clearly points out the interest of using multiple direction measurements in order to weaken, or even remove, conditions upon $x$ and its timevariations. For instance, in the $3 \mathrm{D}$-case $(n=3)$, if $l \geq 2$ then the condition is satisfied provided that the body is periodically not aligned with all the source points. If three or more source points are not aligned, then the condition is automatically satisfied independently of $x$ and its time-variations.

- In the 3D-case, if $l \geq 2$ and the matrix $\Delta(t)$ is positive, and if the body moves with a constant unknown velocity, the above observer provides also an estimation of this velocity. To this aim it suffices to set $u=0$ in the algorithm. The term $\hat{a}$ is then an estimate of the body velocity that is equal to $a$ in this case.

- In the unbiased case where $a=0$ and the body velocity $u$ is measured, the calculation of $\hat{a}$ is superfluous and the above observer reduces to

$$
\dot{\hat{x}}=u-k(t) P(t)\left(\sum_{i=1}^{l} \Pi_{y_{i}(t)} Q_{i i}(t)\left(\hat{x}-z_{i}\right)\right)
$$

with $P(t)$ the solution to the CRE

$$
\dot{P}=-P\left(\sum_{i=1}^{l} \Pi_{y_{i}(t)} Q_{i i}(t) \Pi_{y_{i}(t)}\right) P+V(t)
$$

A particular solution to this latter equation, obtained by choosing $V(t)=\sum_{i=1}^{l} \Pi_{y_{i}(t)} Q_{i i}(t) \Pi_{y_{i}(t)}$, is $P=I_{n \times n}$. To our knowledge, the Riccati observer so obtained has been first derived in [?] in the case of a single source point via a simplified Lyapunov observer design that does not explicitly involve a CRE. This solution is likely not the most effective one, but it is the simplest one.

\section{Position estimation from ranges}

Given the coordinates $z_{i}(i \in\{1, \ldots, l\})$ of $l$ source points expressed in some fixed frame, the problem consists in estimating the position $x$ of a body w.r.t. this frame knowing its velocity $u$ and by using the measurement of the distances (or ranges) $\left|x-z_{i}\right|$ between the body and the source points. For the sake of generality and because this may be useful in practice we propose two possible extensions of the basic observers, namely i) when the measured velocity is biased by an initially unknown constant vector $a$ and ii) when all measured distances are biased by an unknown constant scalar $b$. From these extensions the design of Riccati observers when $a \neq 0$ and $b \neq 0$ poses no difficulty and is left to the interested reader.

\subsection{Unbiased velocity and unbiased range measure- ments}

To simplify the observer design and also prevent the observer equations from being singular when $\left|x-z_{i}\right|=$ 0 , we find it useful to formally set the observer's output $y_{i}$ equal to half the squared distance between the body and the $i t h$ source point, i.e. $y_{i}=0.5\left|x-z_{i}\right|^{2}$, rather than the distance $\left|x-z_{i}\right|$ itself. Define the $(l \times 1)$ dimensional constant vector $\xi:=[1, \ldots, 1]^{\top}$. Define the weighted output variable $y_{0}:=\sum_{i=1}^{l} \alpha_{i}\left(y_{i}-0.5\left|z_{i}\right|^{2}\right)$, with $\alpha=\left[\alpha_{1}, \ldots, \alpha_{l}\right]^{\top}$ denoting a $l$-dimensional vector of real numbers such that $\sum_{i=1}^{l} \alpha_{i}=1$. Since $\dot{x}=u$ and $y_{0}=0.5|x|^{2}-\sum_{i=1}^{l} \alpha_{i} z_{i}^{\top} x$, one has $\dot{y}_{0}=\left(x^{\top}-\right.$ $\left.\sum_{i=1}^{l} \alpha_{i} z_{i}^{\top}\right) u$. Define also the $(l+1)$-dimensional output vector $y:=\left[y_{0},\left(y_{1}-y_{0}-0.5\left|z_{1}\right|^{2}\right), \ldots,\left(y_{l}-y_{0}-\right.\right.$ $\left.\left.0.5\left|z_{l}\right|^{2}\right)\right]^{\top}$ and the augmented state $X:=\left[x^{\top}, y_{0}\right]^{\top} \in$ $\mathbb{R}^{n+1}$. Since $\left(y_{j}-y_{0}-0.5\left|z_{j}\right|^{2}\right)=\sum_{i=1}^{l} \alpha_{i} z_{i}^{\top} x-\alpha_{j} z_{j}^{\top} x$ one has $y=C X$ with

$$
\begin{aligned}
C & :=\left[\begin{array}{cc}
0_{1 \times n} & 1 \\
D(\alpha) Z^{\top} & 0_{l \times 1}
\end{array}\right], D(\alpha):=\xi \alpha^{\top}-I_{l \times l} \\
Z & :=\left[z_{1} \ldots z_{l}\right]
\end{aligned}
$$

Note that $\operatorname{rank}(D(\alpha))=(l-1)$. Using the previous definitions one obtains the linear system

$$
\left\{\begin{array}{l}
\dot{X}=A(t) X+\bar{u} \\
y=C X
\end{array}\right.
$$

$$
\stackrel{\text { with }}{A(t)}:=\left[\begin{array}{cc}
0_{n \times n} & 0_{n \times 1} \\
u^{\top}(t) & 0
\end{array}\right], \bar{u}:=\left[\begin{array}{c}
u \\
-\sum_{i=1}^{l} \alpha_{i}\left(z_{i}^{\top} u\right)
\end{array}\right]
$$

A Riccati observer associated with this system is of the form (15), with $Q(t)$ chosen strictly larger than an arbitrarily small p.d. matrix.

Lemma 4.1 If $u(t)$ and the vectors $z_{i}(i=1, \ldots, l)$ satisfy, for some $\delta>0$ and $\mu>0$, the p.e. condition

$$
\forall t \geq 0: Z D^{\top}(\alpha) D(\alpha) Z^{\top}+\frac{1}{\delta} \int_{t}^{t+\delta} u(s) u^{\top}(s) d s \geq \mu I_{d}
$$

then the above-mentioned Riccati observer globally uniformly exponentially stabilizes $\tilde{X}=0$.

The proof of this lemma again consists in showing that the observability Gramian associated with the system satisfies the property evoked in Lemma 2.6. It is given in Appendix A.4.

In the case of a single source point $D=0$ and the p.e. condition reduces to $\frac{1}{\delta} \int_{t}^{t+\delta} u(s) u^{\top}(s) d s \geq \mu I_{d}>0$, $\forall t \geq 0$. We also remark that the p.e. condition is automatically satisfied, independently of $u(t)$, when $l \geq n+1$ and $\operatorname{rank}\left(D(\alpha) Z^{\top}\right)=n$, i.e. when $n$ vectors among the $(l-1)$ vectors $\left(z_{i}-z_{1}\right), i=2, \ldots, l$, are independent. For instance, in the 3D-case (resp. 2D-case) it is satisfied when four non coplanar (resp. three non-aligned) source points are used. This result is coherent with the minimum number of source points needed to geometrically determine the position of a motionless body with no ambiguity from a single set of multiple range measurements. Using more source points provides redundancy that can be used to accelerate the rate of convergence and/or reduce the asymptotic variance of $\tilde{X}$ when the range measurements are corrupted by noise. 


\subsection{Biased velocity case}

Define

- $X:=\left[x^{\top}, a^{\top}, y_{0}, a^{\top} x,|a|^{2}\right]^{\top}$

- $y:=\left[y_{0},\left(y_{1}-y_{0}-0.5\left|z_{1}\right|^{2}\right), \ldots,\left(y_{l}-y_{0}-0.5\left|z_{l}\right|^{2}\right)\right]^{\top}$, i.e. the same output vector as in the unbiased case;

- $\bar{u}:=\left[u^{\top}, 0_{1 \times n},-\sum_{i=1}^{l} \alpha_{i}\left(z_{i}^{\top} u\right), 0,0\right]^{\top}$.

Forming the time-derivative of $X$ yields a linear system alike (21) with the state and input matrices

$$
\begin{gathered}
A(t)=\left[\begin{array}{ccccc}
0_{n \times n} & I_{n \times n} & 0_{n \times 1} & 0_{n \times 1} & 0_{n \times 1} \\
0_{n \times n} & 0_{n \times n} & 0_{n \times 1} & 0_{n \times 1} & 0_{n \times 1} \\
u^{\top}(t) & -\sum_{i=1}^{l} \alpha_{i} z_{i}^{\top} & 0 & 1 & 0 \\
0_{1 \times n} & u^{\top}(t) & 0 & 0 & 1 \\
0_{1 \times n} & 0_{1 \times n} & 0 & 0 & 0
\end{array}\right] \\
C=\left[\begin{array}{ccccc}
0_{1 \times n} & 0_{1 \times n} & 1 & 0 & 0 \\
D(\alpha) Z^{\top} & 0_{l \times n} & 0_{l \times 1} & 0_{l \times 1} & 0_{l \times 1}
\end{array}\right]
\end{gathered}
$$

A Riccati observer associated with this system is of the form (15), with the matrix $Q(t)$ chosen larger than an arbitrarily small p.d. matrix.

Lemma 4.2 If $u(t)$ is twice differentiable with bounded first and second derivatives, and if $\dot{u}(t)$ and the vectors $z_{i}(i=1, \ldots, l)$ satisfy the p.e. condition

$\forall t \geq 0: Z D^{\top}(\alpha) D(\alpha) Z^{\top}+\frac{1}{\delta} \int_{t}^{t+\delta} \dot{u}(s) \dot{u}^{\top}(s) d s \geq \mu I_{d}$

for some $\delta>0$ and $\mu>0$, then the Riccati observer globally uniformly exponentially stabilizes $\tilde{X}=0$.

The proof of this lemma is given in Appendix A.5. According to this lemma one finds again that the fullrankedness of $D(\alpha) Z^{\top}$ is sufficient to yield the exponential stabilization of $\tilde{X}=0$ independently of the input $u(t)$.

Remark: When the body velocity is constant but unknown a priori, if the matrix $D(\alpha) Z^{\top}$ is of full rank equal to $n$, then the observer provides also an estimate of this velocity. To this aim it suffices to set $u=0$ in the algorithm. The term $\hat{a}$ is then an estimate of the body velocity $a$.

\subsection{Biased range measurements}

A practical reason for considering the case of range measurements corrupted by an additive constant bias $b$ (also called pseudorange measurements) stems from that several range sensors measure times of flight via the use of clocks that are not exactly synchronized. For instance, in the case of GNSS the clocks of the satellites are typically desynchronized from the receiver's clock by a small value $\Delta t$ that produces a range measurement bias equal to $c \Delta t$, with $c$ denoting the speed of light. This leads to estimate $b$ together with the body position. Let us thus assume that the measured distance is $\bar{y}_{i}=\left|x-z_{i}\right|+b(i \in\{1, \ldots, l\})$ with $b$ an unknown real number. By analogy with the unbiased case let us set $y_{i}:=0.5 \bar{y}_{i}^{2}$ for $i \in\{1, \ldots, l\}$ and $y_{0}:=\sum_{i=1}^{l} \alpha_{i}\left(y_{i}-0.5\left|z_{i}\right|^{2}\right)$, with $\alpha=\left[\alpha_{1}, \ldots, \alpha_{l}\right]^{\top}$ denoting a $l$-dimensional vector of real numbers such that $\sum_{i=1}^{l} \alpha_{i}=1$. The (measured) output vector is still $y:=\left[y_{0},\left(y_{1}-y_{0}-0.5\left|z_{1}\right|^{2}\right), \ldots,\left(y_{l}-y_{0}-0.5\left|z_{l}\right|^{2}\right)\right]^{\top}$. Define $\bar{y}:=\left[\bar{y}_{1}, \ldots, \bar{y}_{l}\right]^{\top}$, the augmented state vector as $X:=\left[x^{\top}, y_{0}-b\left(\alpha^{\top} \bar{y}\right), b\right]^{\top} \in \mathbb{R}^{n+2}$ and the input vector $\bar{u}:=\left[u^{\top},-\sum_{i=1}^{l} \alpha_{i}\left(z_{i}^{\top} u\right), 0\right]^{\top}$. One obtains the following LTV system: $\dot{X}=A(t) X+\bar{u}, y=C(t) X$, with

$A(t)=\left[\begin{array}{ccc}0_{n \times n} & 0_{n \times 1} & 0_{n \times 1} \\ u^{\top}(t) & 0 & 0 \\ 0_{1 \times n} & 0 & 0\end{array}\right] C=\left[\begin{array}{ccc}0_{1 \times n} & 1 & \alpha^{\top} \bar{y} \\ D(\alpha) Z^{\top} & 0_{l \times 1} & -D(\alpha) \bar{y}\end{array}\right]$

A Riccati observer associated with this system is of the form (15), with the matrix $Q(t)$ chosen larger than an arbitrarily small p.d. matrix. By application of Lemma 2.4, as for the proofs of Lemmas 4.1 and 4.2, one shows that, in the 3D (resp. 2D) static case using five noncoplanar (resp. four non-aligned) and more source points "generically" ensures global uniform exponential stability of $\tilde{X}=0$. This is coherent with the existence of multiple solutions to the algebraic pseudo-range equations when less source points are used. Moreover, assuming that $|\dot{u}(t)|$ and $|\ddot{u}(t)|$ are bounded, the satisfaction of the p.e. condition (22), complemented with the satisfaction of the condition that $|\dot{\bar{y}}(t)|$ is regularly larger than an arbitrary small positive number, ensures global uniform exponential stability of $\tilde{X}=0$ whatever the number of source points. Note that the first condition is automatically satisfied in the case of four non-coplanar (resp. three non-aligned) and more source points. Note also that the satisfaction of the latter condition implies body motion and that it only requires $|u(t)|$ to be regularly larger than a small positive number in the case of two and more source points. See [?] for a detailed proof of these results.

\section{Simulations}

These simulations illustrate the case of a single source point for which body motion is necessary to grant uniform observability and, subsequently, robust estimation of the body position. The considered source point is located at the origin of the inertial frame and the body moves along a Lissajous curve of equation $x(t)=(20 \cos t-15,20 \sin t,-2 \cos 5 t+6)^{\top}$. One easily verifies that both p.e. conditions (18) and (22) yielding uniform observability when using either direction measurements or range measurements are then satisfied. The body velocity measurement is corrupted by the constant bias $a=(0.33,0.66,0.99)^{\top}$ and initial state conditions are $x(0)=(5,0,4)^{\top}, \hat{x}(0)=(4,6,12)^{\top}$ and $\hat{a}(0)=(0,0,0)^{\top}$. Riccati observers are calculated with $k(t)=1$, as for a Kalman filter, and the corresponding CRE are initialized with $P(0)=100 I_{6}$, when using direction measurements, and $P(0)=100 I_{9}$, when using range measurements. For the matrix $V$ involved in the CRE we have set $V=0.01 \operatorname{diag}\{1,1,1,0,0,0\}+$ $\epsilon_{v} I_{6}$, when using direction measurements, and $V=$ 


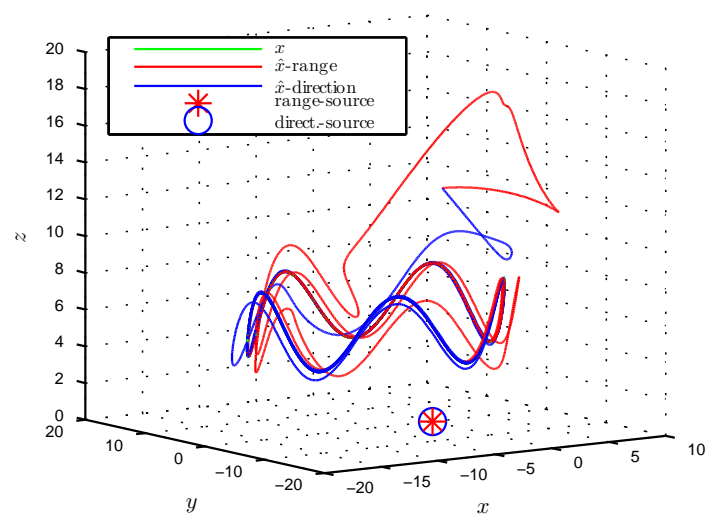

Fig. 1. Trajectories of the body position and its estimates
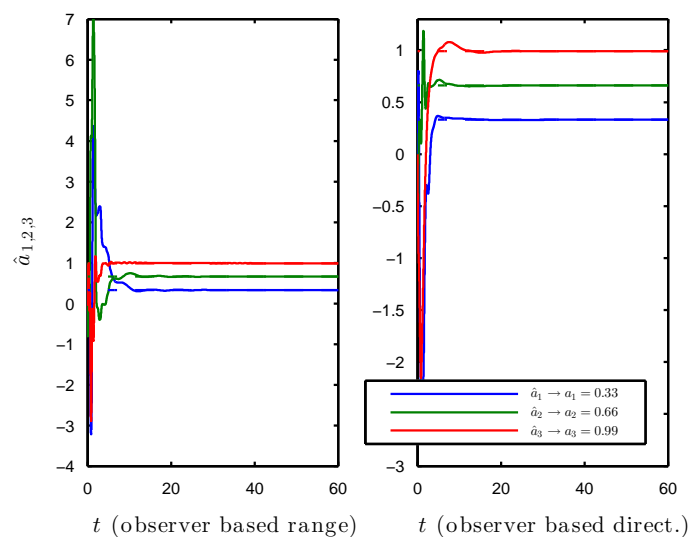

Fig. 2. Time evolution of the velocity bias estimation

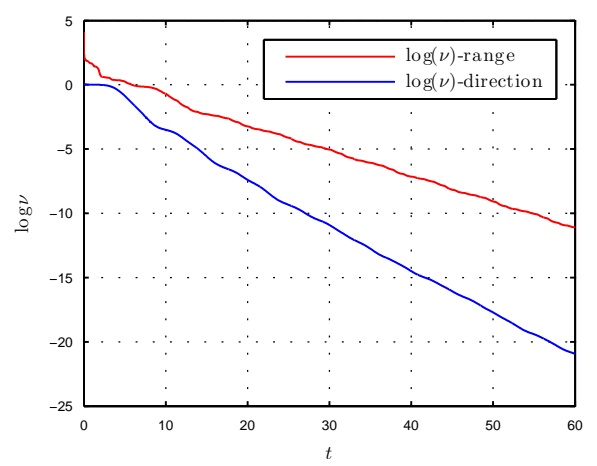

Fig. 3. Time evolution of the Lyapunov functions logarithms

$0.01 \operatorname{diag}\{1,1,1,0,0,0,10,0,0\}+\epsilon_{v} I_{9}$, when using range measurements, with the small number $\epsilon_{v}$ set equal to 0.001 to ensure that the matrix is positive definite. As for the matrix $Q$ we have used $Q=1.5 I_{d}$. Figures $1-3$ illustrate the performance of the two observers in the ideal noise-free case.

\section{Concluding remarks}

In this paper, Riccati observers for the estimation of a body position from either direction or range measurements and from the knowledge of the body velocity have been reviewed. Even when the body velocity is biased by an unknown constant vector, or when the measured distances to the source points are corrupted by a constant bias, these observers ensure global exponential stability of zero estimation errors under uniform observability conditions that have been worked out in relation to the number of source points and the body motion. Clearly the set of such observers extends without difficulty to the case where the available information comes from the combination of direction measurements (associated with certain source points) with range measurements (associated with other source points). A logical prolongation of this work is the derivation of Riccati observers for the estimation of the complete body pose (position and orientation). We foresee several other possible extensions. Let us just mention vision-based robotic applications involving the control of the body position from estimates provided by Riccati observers, and a deterministic approach to Simultaneous Localication and Mapping (SLAM) that could usefully complement existing studies on the subject.

\section{A Appendices}

A.1 Ultimate bounds for $p_{m}$ and $p_{M}$ when $v_{m}>0$ and $W_{Q}(t, t+\delta) \geq \mu I_{d}>0$

Denote the suprema of the spectral norm of $A(t)$ and of $\operatorname{tr}\left(C^{\top}(t) Q(t) C(t)\right)$ as $k_{a}$ and $\bar{\mu}_{q}$ respectively. In [?] we derive the following bounds

$$
\begin{gathered}
\liminf _{t \rightarrow \infty} \lambda_{\min }(P(t)) \geq \frac{v_{m}}{n k_{a}}\left(1+\left(1+\frac{\bar{\mu}_{q} v_{m}}{n k_{a}^{2}}\right)^{0.5}\right)^{-1} \\
\limsup _{t \rightarrow \infty} \lambda_{\max }(P(t)) \leq \frac{1}{\mu \delta}+\frac{1}{3}\left(\frac{\bar{\mu}_{q}}{\mu}\right)^{2} \exp \left(6 k_{a} \delta\right) \delta v_{M}
\end{gathered}
$$

For the latter bound we have used the expression of the upper bound of $P(t)$ derived in [?]. These bounds can in turn be used to estimate an ultimate lower bound of $\frac{p_{m}}{p_{M}^{2}} v_{m}$, i.e. an estimate of the lower bound pointed out in (9) of the exponential rate of convergence associated with a Riccati observer.

\section{A.2 Proof of Lemma 2.7}

Let us proceed by contradiction and assume that the lemma's conclusion is wrong, i.e.

$$
\forall \epsilon, \forall \bar{\delta}>0, \exists t \geq 0: W_{A, H}^{\Sigma}(t, t+\bar{\delta})<\epsilon I_{d}
$$

Consider a sequence $\left\{\epsilon_{p}\right\}_{p \in \mathbb{N}}$ of positive numbers converging to zero, and an arbitrary positive number $\bar{\delta}$. From the previous assertion there must exist a sequence of time-instants $\left\{t_{p}\right\}_{p \in \mathbb{N}}$ and a sequence $\left\{x_{p}\right\}_{p \in \mathbb{N}}$ with $x_{p} \in S^{n-1}$ (i.e. $\left|x_{p}\right|=1$ ) such that $\forall p \in \mathbb{N}: x_{p}^{\top} W_{A, H}^{\Sigma}\left(t_{p}, t_{p}+\bar{\delta}\right) x_{p}<\epsilon_{p}$. Since $S^{n-1}$ is a compact set there exists a sub-sequence of $\left\{x_{p}\right\}_{p \in \mathbb{N}}$ which converges to a limit $\bar{x} \in S^{n-1}$. Therefore 


$$
\lim _{p \rightarrow \infty} \bar{x}^{\top} W_{A, H}^{\Sigma}\left(t_{p}, t_{p}+\bar{\delta}\right) \bar{x}=0
$$

Let $\Sigma^{\frac{1}{2}}(t)$ denote the p.s.d square root of $\Sigma(t)$. The previous equality is the same as

$$
\lim _{p \rightarrow \infty} \int_{0}^{\bar{\delta}}\left|\Sigma^{\frac{1}{2}}\left(t_{p}+s\right) H \exp (A s) \bar{x}\right|^{2} d s=0
$$

which in turn implies

$$
\lim _{p \rightarrow \infty} \int_{\bar{\delta}-\delta}^{\bar{\delta}}\left|\Sigma^{\frac{1}{2}}\left(t_{p}+s\right) H \exp (A s) \bar{x}\right|^{2} d s=0
$$

provided that $\bar{\delta} \geq \delta$. The p.e. condition (12) also implies that

$$
\forall t: \frac{1}{\delta} \int_{t}^{t+\delta} \Sigma^{\frac{1}{2}}(s) d s \geq \epsilon I_{d}>0
$$

for some $\epsilon>0$. This $\epsilon$ number is used in what follows. Consider now the following technical result whose proof is given at the end of the present appendix

Lemma A.1 Assume that the eigenvalues of the matrix $A$ are all real, then, given $\bar{x} \in S^{n-1}$, there exist $r \geq 0$, $\lambda \in \mathbb{R}$, and $z \in \mathbb{R}^{m}-\{0\}$ such that $\frac{\operatorname{Hexp}(A t) \bar{x}}{t^{r} \exp (\lambda t)}=z+\eta(t)$ with $\lim _{t \rightarrow+\infty} \eta(t)=0$.

In view of this result, setting $\bar{z}=z /|z| \in S^{m-1}$, and choosing $\bar{\delta}$ large enough so that $\sup _{s \in[\bar{\delta}-\delta, \bar{\delta}]}|\eta(s)|<$ $\sqrt{\frac{\epsilon}{2}}|z|$ one deduces that

$$
\begin{aligned}
& \frac{1}{\gamma^{2}|z|^{2}} \int_{\bar{\delta}-\delta}^{\bar{\delta}}\left|\Sigma^{\frac{1}{2}}\left(t_{p}+s\right) H \exp (A s) \bar{x}\right|^{2} d s \\
& \geq \int_{\bar{\delta}-\delta}\left|\Sigma^{\frac{1}{2}}\left(t_{p}+s\right)\left(\bar{z}+\frac{\eta(s)}{|z|}\right)\right|^{2} d s \\
& \left.\geq \int_{t_{p}+\bar{\delta}-\delta}^{t_{p}+\bar{\delta}}\left|\Sigma^{\frac{1}{2}}(s) \bar{z}\right|^{2} d s-\int_{0}^{\bar{\delta}} \mid \Sigma^{\frac{1}{2}}\left(t_{p}+s\right) \frac{\eta(s)}{|z|}\right)\left.\right|^{2} d s \\
& \geq \delta \epsilon-\delta \epsilon / 2(=\delta \epsilon / 2)
\end{aligned}
$$

with $\gamma=i n f_{s \in[\bar{\delta}-\delta, \bar{\delta}]}\left(s^{r} \exp (\lambda s)\right)>0$. Therefore

$$
\int_{\bar{\delta}-\delta}^{\bar{\delta}}\left|\Sigma^{\frac{1}{2}}\left(t_{p}+s\right) H \exp (A s) \bar{x}\right|^{2} d s \geq \gamma^{2}|z|^{2} \frac{\epsilon}{2}>0
$$

Since this latter inequality holds true for any $t_{p}$, it contradicts (A.3) and the initial assumption according to which the result of the lemma is not true.

It only remains to prove the technical Lemma A.1. From Cayley-Hamilton's theorem, one has $\exp (A t)=$ $\sum_{i=0}^{n-1} \alpha_{i}(t) A^{i}$ with $\alpha_{i}(t)=\sum_{k=1}^{d}\left(\sum_{j=0}^{l_{k}-1} a_{i j} t^{j}\right) \exp \left(\lambda_{k} t\right)$, $\lambda_{k}$ a (real) eigenvalue of $A, a_{i j} \in \mathbb{R}, d \leq n$ the number of distinct eigenvalues, and $l_{k}$ the multiplicity of $\lambda_{k}$. Therefore

$$
\begin{aligned}
H \exp (A t) \bar{x} & =H \sum_{i=0}^{n-1} \alpha_{i}(t) A^{i} \bar{x}=\sum_{i=0}^{n-1} \alpha_{i}(t) H A^{i} \bar{x} \\
& =\sum_{i=0}^{n-1} \alpha_{i}(t) z_{i}
\end{aligned}
$$

with $z_{i}:=H A^{i-1} \bar{x}$. The (Kalman) observability of the pair $(A, H)$ implies that at least one of the $z_{i}$ vectors is different from zero. The previous sum can also be arranged as follows

$$
\sum_{i=0}^{n-1} \alpha_{i}(t) z_{i}=\sum_{k, j} v_{k, j}(t) \bar{z}_{k, j} \quad \bar{z}_{k, j} \in \mathbb{R}^{m}
$$

with $v_{k, j}(t)=t^{r_{k, j}} \exp \left(\lambda_{k} t\right), k \in[1, \ldots, n], r_{k, j} \in$ $[0, \ldots, n-1]$. At least one of the vectors $\bar{z}_{k, j}$ is different from zero. Consider the largest (less negative, or most positive) root $\lambda_{k}$ for which $\bar{z}_{k, j}$ is different from zero, and the largest power $r_{k, j}$ that goes with such a vector. Denote this root as $\lambda$ and this power as $r$, set $v(t):=t^{r} \exp (\lambda t)$, and denote the corresponding vector $\bar{z}_{k, j}$ as $z(\neq 0)$. The dominating coefficient in the development of $H \exp (A t) \bar{x}$, when $t$ tends to infinity, is thus $v(t)$ and one has $\lim _{t \rightarrow \infty} \frac{H \exp (A t) \bar{x}}{v(t)}=z$. This latter property can also be written as $\frac{H \exp (A t) \bar{x}}{v(t)}=z+\eta(t)$ with $\lim _{t \rightarrow \infty} \eta(t)=0$.

A.3 Proof of Lemma 3.1

Using the expressions of $C(t)$ and $Q(t)$ one has

$$
C^{\top}(s) Q(s) C(s)=\left[\begin{array}{cc}
\Sigma(s) & 0_{n \times n} \\
0_{n \times n} & 0_{n \times n}
\end{array}\right]=H^{\top} \Sigma(s) H
$$

with $\Sigma(s):=\sum_{i=1}^{l} \Pi_{y_{i}(s)} Q_{i i}(s) \Pi_{y_{i}(s)}$ and $H \quad:=$ $\left[\begin{array}{cc}I_{d} & 0_{n \times n}\end{array}\right]$. Therefore

$$
\begin{aligned}
W_{Q}(t, t+\delta) & =\frac{1}{\delta} \int_{t}^{t+\delta} \Phi^{\top}(s, t) C^{\top}(s) Q C(s) \Phi(s, t) d s \\
& =\frac{1}{\delta} \int_{t}^{t+\delta} \Phi^{\top}(s, t) H^{\top}(s) \Sigma(s) H \Phi(s, t) d s
\end{aligned}
$$

with $\Phi(t, s)=\exp (A(s-t))$. Note that the p.e. condition in the lemma implies the existence of $\bar{\epsilon}>0$ such that the condition (12) in Lemma 2.7 holds true. Moreover, the matrix $A$ is constant and its eigenvalues are all equal to zero. They are thus real. One also easily verifies that the pair $(A, H)$ is Kalman observable. Therefore, by application of Lemma 2.7, the observability Gramian $W_{Q}(t, t+\delta)$ associated with the system is strictly larger than some positive constant, $\forall t$. The lemma's conclusion follows immediately.

\section{A.4 Proof of Lemma 4.1}

Recalling that the positivity of the observability Gramian $W$ yields the positivity of the Riccati observability Gramian $W_{Q}$ when $Q(t) \geq \epsilon I_{d}>0$, one only has to show -according to Lemma 2.4- the existence of an adequate matrix-valued function $M($.$) that satisfies (3)$ for some positive numbers $\bar{\delta}$ and $\bar{\mu}$. Define

$$
M(t):=\left[\begin{array}{c}
N_{0} \\
\bar{N}_{1}(t)
\end{array}\right]=\left[\begin{array}{cc}
0_{1 \times n} & 1 \\
D(\alpha) Z^{\top} & 0_{l \times 1} \\
u^{\top}(t) & 0
\end{array}\right]
$$

with $N_{0}=C$ and $\bar{N}_{1}(t)$ the first line of $N_{1}(t)=C A(t)$. Then

$$
M^{\top}(t) M(t)=\left[\begin{array}{cc}
Z D^{\top}(\alpha) D(\alpha) Z^{\top}+u u^{\top} & 0_{n \times 1} \\
0_{1 \times n} & 1
\end{array}\right]
$$

and the result follows directly.

\section{A.5 Proof of Lemma 4.2}

As in the unbiased case we show the existence of a matrix-valued function $M($.$) that satisfies (3) for some$ positive numbers $\bar{\delta}$ and $\bar{\mu}$. Define 


$$
M(t):=\left[\begin{array}{c}
N_{0} \\
N_{1}(t) \\
N_{2}(t)
\end{array}\right]
$$

with $N_{0}=C, N_{1}(t)=C A(t), N_{2}(t)=N_{1}(t) A(t)+$ $\dot{N}_{1}(t)$. Using the expressions of $A(t)$ and $C$ one verifies that

$$
M(t)=\left[\begin{array}{ccccc}
0_{1 \times n} & 0_{1 \times n} & 1 & 0 & 0 \\
D(\alpha) Z^{\top} & 0_{l \times n} & 0_{l \times 1} & 0_{l \times 1} & 0_{l \times 1} \\
u^{\top}(t) & -\sum_{i=1}^{l} \alpha_{i} z_{i}^{\top} & 0 & 1 & 0 \\
0_{l \times n} & D(\alpha) Z^{\top} & 0 & 0 & 0 \\
\dot{u}(t)^{\top} & 2 u^{\top}(t) & 0 & 0 & 1
\end{array}\right]
$$

Consider an arbitrary unit vector $b=\left[b_{1}^{\top}, b_{2}^{\top}, b_{3}, b_{4}, b_{5}\right]^{\top} \in$ $\mathbb{R}^{2 n+3}$, with $b_{1-5}$ denoting sub-vectors of adequate dimensions. Then

$$
M(t) b=\left[\begin{array}{c}
b_{3} \\
D(\alpha) Z^{\top} b_{1} \\
u^{\top}(t) b_{1}-\sum_{i=1}^{l} \alpha_{i} z_{i}^{\top} b_{2}+b_{4} \\
D(\alpha) Z^{\top} b_{2} \\
\dot{u}^{\top}(t) b_{1}+2 u^{\top}(t) b_{2}+b_{5}
\end{array}\right]
$$

and $|M(t) b|^{2}=b_{3}^{2}+b_{1}^{\top} Z D D^{\top} Z b_{1}+\left(u^{\top}(t) b_{1}-\right.$ $\left.\sum_{i=1}^{l} \alpha_{i} z_{i}^{\top} b_{2}+b_{4}\right)^{2}+b_{2}^{\top} Z D D^{\top} Z b_{2}+\left(\dot{u}^{\top}(t) b_{1}+\right.$ $\left.2 u^{\top}(t) b_{2}+b_{5}\right)^{2}$. Define $\gamma(t):=\frac{1}{\delta} \int_{t}^{t+\delta}|M(s) b|^{2} d s$ and let us make a proof by contradiction by assuming that the condition (3) is not satisfied. In this case there exists a sequence $\left\{t_{p}\right\}$ and a unit vector $b \in \mathbb{R}^{2 n+3}$ such that $\lim _{p \rightarrow+\infty} \gamma\left(t_{p}\right)=0$. This in turn implies that $b_{3}=b_{1}^{\top} Z D D^{\top} Z b_{1}=b_{2}^{\top} Z D D^{\top} Z b_{2}=0$ and also

$$
\begin{aligned}
& \lim _{p \rightarrow+\infty} \int_{t_{p}}^{t_{p}+\delta}\left(u^{\top}(t) b_{1}-\sum_{i=1}^{l} \alpha_{i} z_{i}^{\top} b_{2}+b_{4}\right)^{2} d s=0 \\
& \lim _{p \rightarrow+\infty} \int_{t_{p}}^{t_{p}+\delta}\left(\dot{u}^{\top}(t) b_{1}+2 u^{\top}(t) b_{2}+b_{5}\right)^{2} d s=0
\end{aligned}
$$

Using the assumed boundedness of $\dot{u}(t)$ the first of these two limits yields $\lim _{p \rightarrow+\infty} u^{\top}\left(t_{p}+s\right) b_{1}=$ $-b_{4}+\sum_{i=1}^{l} \alpha_{i} z_{i}^{\top} b_{2}, \forall s \in(0, \delta)$. Using now the assumed boundedness of $\ddot{u}(t)$ this in turn implies that $\lim _{p \rightarrow+\infty} \dot{u}^{\top}\left(t_{p}+s\right) b_{1}=0, \forall s \in(0, \delta)$. From (A.5) one deduces that $\lim _{p \rightarrow+\infty}\left(\dot{u}^{\top}\left(t_{p}+s\right) b_{1}+2 u^{\top}\left(t_{p}+\right.\right.$ $\left.s) b_{2}+b_{5}\right)=0, \forall s \in(0, \delta)$ and, subsequently that $\lim _{p \rightarrow+\infty} \dot{u}^{\top}\left(t_{p}+s\right) b_{2}=-b_{5} / 2, \forall s \in(0, \delta)$. Using the assumed boundedness of $\ddot{u}(t)$ this in turn implies that $\lim _{p \rightarrow+\infty} \dot{u}^{\top}\left(t_{p}+s\right) b_{2}=0, \forall s \in(0, \delta)$. Therefore $\lim _{p \rightarrow+\infty} b_{1}^{\top}\left(\dot{u}\left(t_{p}+s\right) \dot{u}\left(t_{p}+s\right)^{\top}+\delta Z D D^{\top} Z\right) b_{1}=0$ and $\lim _{p \rightarrow+\infty} b_{2}^{\top}\left(\dot{u}\left(t_{p}+s\right) \dot{u}\left(t_{p}+s\right)^{\top}+\delta Z D D^{\top} Z\right) b_{2}=0$, $\forall s \in(0, \delta)$. In view of the p.e. condition (23) this in turn implies that $b_{1}=b_{2}=0$. Since $b_{3}$ is also equal to zero, the convergence of $\gamma\left(t_{p}\right)$ to zero when $p$ tends to infinity necessarily implies that $b_{4}^{2}+b_{5}^{5}=0$ so that $b=0$. This is not possible since, by assumption, $b$ is a unit vector. This contradiction thus implies that $M(t)$ satisfies the condition (3).

\section{Acknowledgements}

This work was supported by the ANR-ASTRID project SCAR "Sensory Control of Unmanned Aerial Vehicles", the ANR-Equipex project "Robotex".

\section{References}

[1] D. Aeyels, R. Sepulchre, and J. Peuteman. Asymptotic stability for time-variant systems and observability: uniform and nonuniform criteria. Mathematics of Control, Signals and Systems, 11(1):1-27, 1998.

[2] P. Batista, C. Silvestre, and P. Oliveira. Single range navigation in the presence of constant unknown drifts. In European Control Conference (ECC), pages 3983-3998, 2009.

[3] P. Batista, C. Silvestre, and P. Oliveira. Single range aided navigation and source localization: observability and filter design. Systems \& Control Letters, 60:665-673, 2011.

[4] P. Batista, C. Silvestre, and P. Oliveira. Globally exponentially stable filters for source localization and navigation aided by direction measurements. Systems $\&$ Control Letters, 62(11):1065-1072, 2013.

[5] P. Batista, C. Silvestre, and P. Oliveira. Sensor-based long baseline navigation: observability analysis and filter design. Asian J. Control, 16(4):974-994, 2014.

[6] P. Batista, C. Silvestre, and P. Oliveira. Navigation systems based on multiple bearing measurements. IEEE Transactions on Aerospace and Electronic Systems, 51(4):2887-2899, 2015.

[7] G. Besançon. An overview on observer tools for nonlinear systems. In Nonlinear Observers and Applications, Besançon G., Editor, Lecture Notes in Control and Information Sciences, 363, Springer-Verlag, 2007.

[8] F. Le Bras, T. Hamel, R. Mahony, and C. Samson. Observer design for position and velocity bias estimation from a single direction output. In 54th IEEE International Conference on Decision and Control (CDC), 2015.

[9] C.-T. Chen. Linear System Theory and Design. CBS College Publishing, second edition, 1984.

[10] S. H. Dandach, B. Fidan, S. Dasgupta, and B.D.O. Anderson. A continuous time linear adaptive source localization algorithm, robust to persistent drift. Systems $\&$ Control Letters, 58(1):7-16, 2009.

[11] T.H. Dixon. An introduction to the global positioning system and some geological applications. Reviews of geophysics, 29(2):249-276, 1991.

[12] J.-P. Gauthier and J.-P. Kupka. Observability and observers for nonlinear systems. SIAM J. on Control Optim., 4(32):975-994, 1994.

[13] T. Hamel and C. Samson. Riccati observers for position and velocity bias estimation from either direction or range measurements. Technical Report arXiv:1606.07735, 2016.

[14] M. Pengov, E. Richard, and J.-C. Vivalda. On the boundedness of the solutions of the continuous riccati equation. J. of Inequal. and Appli., 6:641-649, 2001.

[15] G. Scandaroli. Fusion de données visuo-inertielles pour l'estimation de pose et l'autocalibrage. PhD thesis, University of Nice Sophia-Antipolis, France, HAL Id: tel$00861858,2013$. 